\title{
An estimate of the DM profile in the Galactic bulge region
}

\author{
Fabio Iocco*, Maria Benito \\ ICTP South American Institute for Fundamental Research, and Instituto de Física Teórica - Universidade Estadual Paulista (UNESP), Rua Dr. Bento Teobaldo \\ Ferraz 271, 01140-070 São Paulo, SP, Brazil
}

\section{A R T I C L E I N F O}

\section{Article history:}

Received 14 October 2016

Received in revised form 19 December 2016

Accepted 19 December 2016

\section{Keywords:}

Dark Matter

Milky Way

Galactic Center

Indirect detection

Stellar dynamics

\begin{abstract}
A B S T R A C T
We present an analysis of the mass distribution in the region of the Galactic bulge, which leads to constraints on the total amount and distribution of Dark Matter (DM) therein. Our results - based on the dynamical measurement of the BRAVA collaboration - are quantitatively compatible with those of a recent analysis, and generalized to a vast sample of observationally inferred morphologies of the stellar components in the region of the Galactic bulge. By fitting the inferred DM mass to a generalized NFW profile, we find that cores $(\gamma \lesssim 0.6)$ are forbidden only for very light configurations of the bulge, and that cusps $(\gamma \gtrsim 1.2)$ are allowed, but not necessarily preferred. Interestingly, we find that the results for the bulge region are compatible with those obtained with dynamical methods (based on the rotation curve) applied to outer regions of the Milky Way, for all morphologies adopted. We find that the uncertainty on the shape of the stellar morphology heavily affects the determination of the DM distribution in the bulge region, which is gravitationally dominated by baryons, adding up to the uncertainty on its normalization. The combination of the two hinders the actual possibility to infer sound conclusions about the distribution of DM in the region of the Galactic bulge, and only future observations of the stellar census and dynamics in this region will bring us closer to a quantitatively more definite answer.
\end{abstract}

(c) 2016 Elsevier B.V. All rights reserved.

\section{Introduction}

The density structure of the Dark Matter halo embedding our own Galaxy, the Milky Way (MW), is the object of a continuous research effort, which has its roots in several decades of rigorous astronomical activity. The best known method for deriving the total mass structure of the MW is probably that based on the use of tracers of the Rotation Curve (RC), which in turn traces the total gravitational potential in galaxies which - like our own - are rotation supported. Once the total mass profile is inferred through this method, one can in principle subtract that of the visible component (obtained through independent observations), and fit the extracted residuals to pre-assigned spherical shapes, referred to as DM density profiles. Examples of the application of this method can be found in [1-4], and a review of the history and results of such method, often referred to as "global", can be found in [5]. This technique does however rely on the assumption of circular orbits for the tracers of the RC, and an axisymmetric gravitational potential, and therefore cannot be adopted in regions where either one of the two assumptions is broken, namely at galactocentric distances $R \lesssim 2.5 \mathrm{kpc}$ (or, under more conservative approach $R \lesssim 4 \mathrm{kpc}$, [6]). Alternative methods, either based on a

\footnotetext{
* Corresponding author.

E-mail addresses: fabio.iocco.astro@gmail.com (F. Iocco), mariabenitocst@gmail.com (M. Benito).
}

different approach like the one adopted in [7], or in the release of the ansatz on the shape of the distribution like the one adopted in [8], also fail to address the region of the Galactic bulge.

Whereas one does expect the DM to be a subleading component of the matter content in this central region of the Galaxy, our forced ignorance about it still remains frustrating. In fact, the actual content and density structure of DM in such a small region (of the MW, and of other galaxies) is at the center of a long-standing debate on weather the DM profile of galaxies is "cored" or "cusped", see e. g. [9] which has been recently revived by the vigorous developments in numerical simulations of Galaxy formation in cosmological environments, e. g. [10-12], with the interesting finding of a DM profile which responds to the presence of baryons, and exhibits a core or a cusp profile in different regimes of the stellar over halo mass, $[13,14]$. The DM profile in the GC region is also a very important ancillary quantity for "indirect" searches, which often target regions of supposedly higher DM density, like the Galactic Center, thus making this quantity of high relevance also in the search for the very nature of the DM, e.g. [15,16].

In order to obtain an estimate of the DM mass and density profile (and related uncertainties) in the region of the Galactic bulge, we follow here the rationale of the recent analysis in [17]. We adopt an estimate of the dynamical mass within a small region around the Galactic center [18,19], obtained through observations of the BRAVA survey $[20,21]$. Separately, we estimate the stellar 
mass in the same region by making use of a vast sample of observationally inferred stellar morphologies, and fit the residuals of the two to a generalized NFW profile (gNFW). Owing to a larger sample of stellar morphologies, and a most conservative estimate of the statistical uncertainty on the stellar mass, our results - while remaining compatible with those in [17] - show a much larger band of uncertainty for the slope of the profile, $\gamma$, and remain yet inconclusive.

\section{Method}

We first adopt a determination of the total mass in the region of interest, a square box of coordinates around the Galactic Center:

$[x, y, z]=[ \pm 2.2, \pm 1.4, \pm 1.2]$;

which reads $M_{\text {total }}=(1.84 \pm 0.07) \times 10^{10} M_{\odot}$.

This estimate is presented in [18], after a reconstruction of the recent 3D density of red clump giants (RCG) as well as kinematic measurements, from the BRAVA survey [20,21], and adopted in [17]. ${ }^{1}$

It is interesting to notice that the extremes of the region in exam are complementary to the validity limit of analysis based on dynamical tracers, which as discussed are typically restricted to galactocentric radii $R>2.5 \mathrm{kpc}$. In order to know the DM content, we now need information about the stellar mass in the very same region, so as to obtain the component missing from the total gravitational budget.

The estimate of the stellar (baryonic) mass in the region of interest, is where our analysis most diverges from that in [17]: rather than relying on the results of [18], based on $N$-body, madeto-measure simulations implementing a peanut-shaped bulge and different bulge-to-disc ratio - resulting in five different morphologies for the region of interest -, we adopt a wider array of bulge (and disc) shapes modeled and normalized after observational data, all available in the literature.

This collection of three-dimensional morphologies (see Tables for references) has been first presented in [22] and later adopted in $[4,8]$ in order to estimate the ignorance on the actual morphology and total mass of the baryonic component of the MW, comparing the existing stellar distribution profiles. In order to obtain the baryonic mass, we integrate the three dimensional stellar density $\rho_{*}^{i}(R, \theta, \phi)$ thus collected (where the index $i$ runs over the morphology type) over the region of interest. At no point we do perform any average or mix the information of different bulges (nor discs): we simply present the information available for all the morphologies in the literature in order to represent at best the current uncertainty, thus offering an estimate of the current systematics. The normalization of the profiles is given for all of them in the source reference, however for most cases uncertainties are not available. In order to obtain a reliable estimate on the uncertainty on the stellar mass, and at the same time to comply with existing constraints on microlensing, we normalize the stellar mass distribution of the bulge by imposing the MACHO constraints on microlensing optical depth toward the region of the Galactic Center, [23]. This technique has been first described in [2], and widely adopted in the literature after then. For the stellar component in the $\operatorname{disc}(\mathrm{s})$, we adopt a normalization based on a recent estimate of the surface stellar density, [7]. We address the reader to the original references and to [4] for a thorough description of these techniques and a test of their validity, and we stress here that the existing observational uncertainty on the microlensing optical depth and disc stellar surface density automatically assigns

\footnotetext{
1 We have explicitly tested that our results do not change if we adopt the updated estimate of total dynamical mass, $M_{\text {total }}=(1.85 \pm 0.05) \times 10^{10} M_{\odot}$, presented by the same authors in [19].
}

a (statistically meaningful) uncertainty to the stellar mass, which becomes thus comparable (and compared) to that of the total mass, as inferred by the dynamical measurement of the BRAVA survey. It is worth stressing that the bulge normalization is indeed affected by the stellar disc adopted: we do also account for that - it is the reason of the different bulge masses between the different panels of the Tables - however the choice of the disc does not change our conclusions. We will however present self-consistent results for all bulges and disc morphologies in our sample. The Galaxy also contains a disc of diffuse, interstellar gas [24,25] which we neglect here, because its contribution to the total baryonic mass in this region is below the smallest of uncertainties in our budget.

The procedure described above permits to derive the DM mass allowed in the region of interest, and its uncertainty, as a simple difference between the observational derivation of the total mass $M_{\text {tot }}$, and the inference of the stellar mass from observationally inspired profiles $M_{*}^{i}$, where the index $i$ runs over all the possible combination of bulges and discs.

For each stellar morphology in our sample we can now obtain an allowed DM mass (and its uncertainty), simply as the difference between $M_{\text {tot }}$ and $M_{*}^{i}$ (thus the allowed DM mass will be a function of the adopted morphology), and at this point we set out to understand what constraints does this impose on the parameters of a generalized NFW (gNFW) profile:

$\rho_{D M}(R)=\rho_{0}\left(\frac{R_{0}}{R}\right)^{\gamma}\left(\frac{R_{S}+R_{0}}{R_{S}+R}\right)^{3-\gamma}$,

by identifying the regions in the $\left(\rho_{0}, \gamma, R_{s}\right)$ space that would bear a mass in excess or defect with respect to the one allowed, per each morphology.

It is to be noticed that - as the methodology described is self consistently defined within a small region around the Galactic Center - the meaning of the index $\gamma$ is purely local, and one can see it as an effective index valid only in the region described.

\section{Results}

Finally, we present here our results, displaying in all Figures the regions in the gNFW parameter space which produce a mass compatible with that allowed by procedure described above, within one sigma (in white), within one and two sigmas (pale pink), and in tension at more than two sigmas (dark purple). We first show the results varying the bulge morphology (which sets most of the stellar mass in the region of interest), and by keeping constant Galactic parameters $\left(R_{0}, v_{0}\right)=(8 \mathrm{kpc}, 230 \mathrm{~km} / \mathrm{s})$ and disc component. Fig. 1 displays the results for our six bulge configurations, in the $\left(\rho_{0}, \gamma\right)$ plane, for an assigned scale radius $R_{s}=20 \mathrm{kpc}$.

The effect of the variation of scale radius is indeed small, as it can be seen in Fig. 2, where we show the constraints in the same $\left(\rho_{0}, \gamma\right)$ plane for three different values of $R_{s}$, spanning the extremes of the range suggested by numerical simulations; we therefore keep the choice of $R_{S}=20 \mathrm{kpc}$ for the following plots. We also check the effect of varying the Sun's Galactocentric distance $R_{0}$ within the allowed range $R_{0}=[7.5,8.5] \mathrm{kpc}$ (notice that the technique adopted here is - unlike the RC method - insensitive to the value of the local circular velocity $v_{c}$ ), the results can be seen in Fig. 3, and motivate us to keep a constant $R_{0}=8 \mathrm{kpc}$ for the rest of this paper.

As it can be easily noticed, accounting for uncertainties in the determination of the stellar mass opens up a relatively ample region in the parameter space. While not prohibiting extremely peaked profiles, our analysis shows no real preference for a cuspy DM distribution in the region of interest, the lowest values of $\gamma$ being disfavored for few configurations only.

In Fig. 4, we show the effects of different discs and bulges, thus encompassing effectively all possible morphologies. 

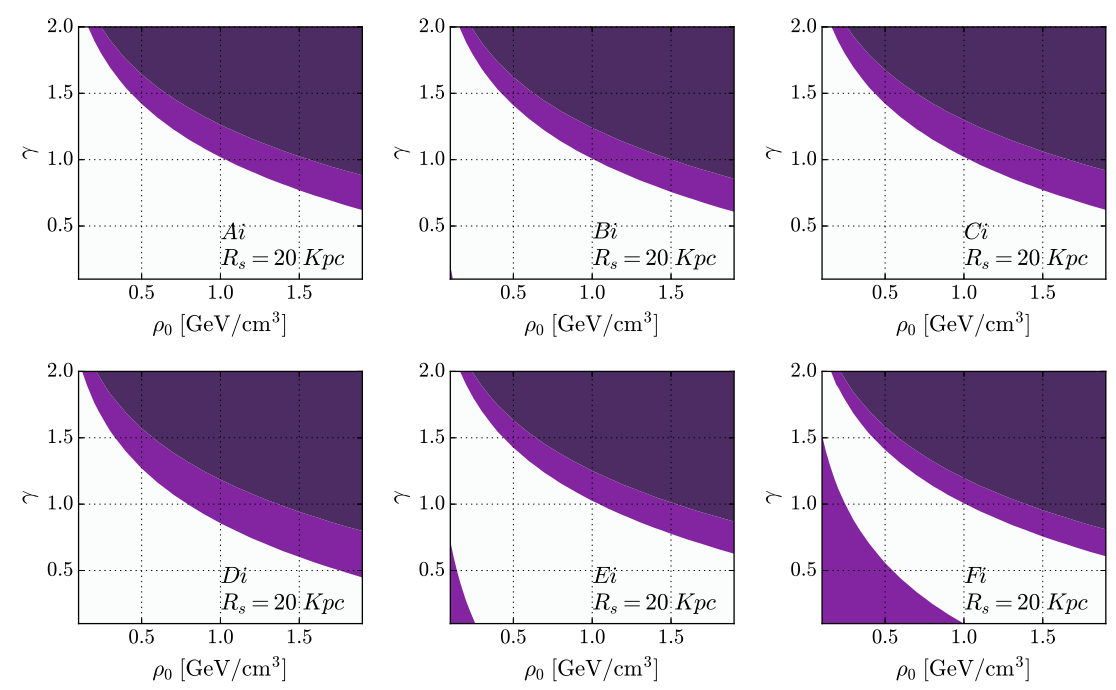

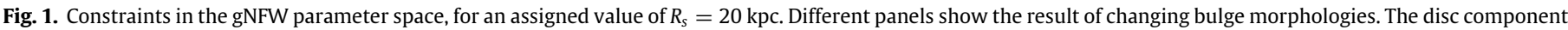
is fixed to [26]. (For interpretation of the references to color in this figure legend, the reader is referred to the web version of this article.)
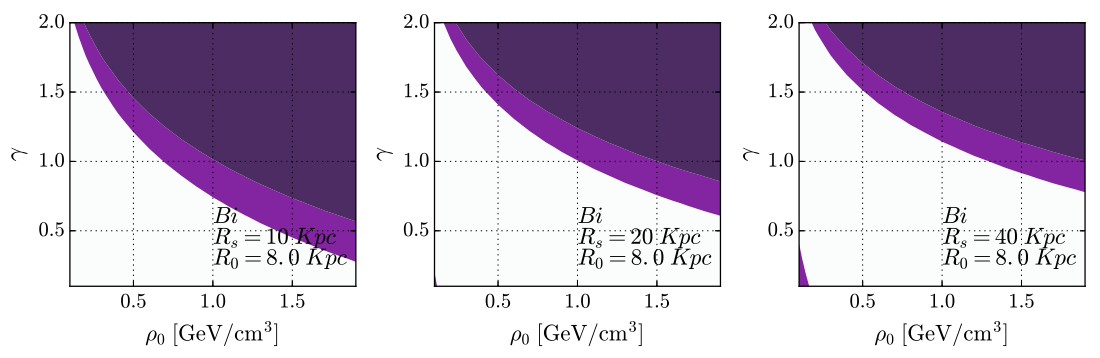

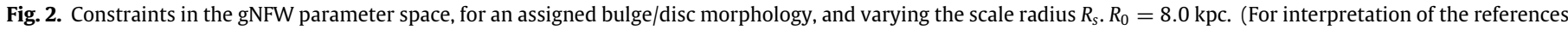
to color in this figure legend, the reader is referred to the web version of this article.)
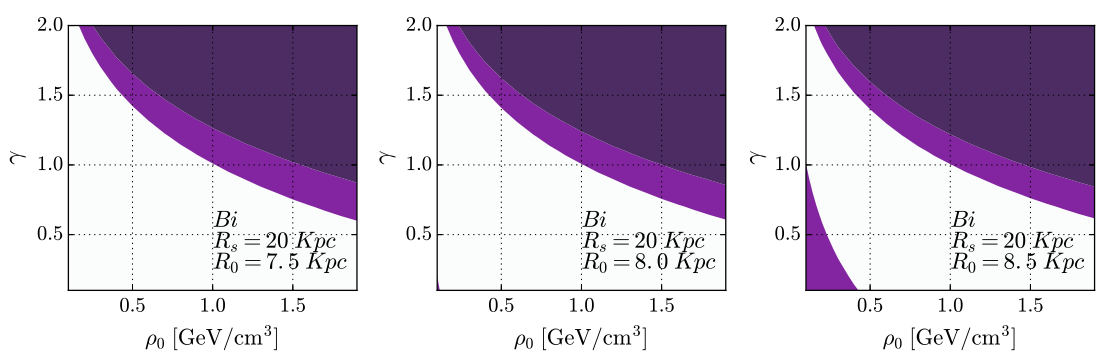

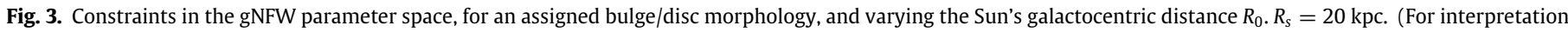
of the references to color in this figure legend, the reader is referred to the web version of this article.)

In these plots, we have also shown - for each baryonic morphology - preferred regions from the RC analysis in [4], picking the 99.73\% $(3 \sigma)$ confidence region encompassed by $\chi^{2} \leq \chi_{\mathrm{bf}}^{2}+\Delta \chi^{2}$, where $\chi_{\mathrm{bf}}^{2}$ is the $\chi^{2}$ of the best fit configuration and $\Delta \chi^{2}=11.83$ (corresponding to two fitted parameters at $3 \sigma$ ). While performing this comparison, one has to bear in mind that the region studied by the two analysis (which are also different in methodologies) are complementary. The index $\gamma$ obtained by the RC analysis is based on a comparison of the tracers of the total gravitational potential at $R>2.5 \mathrm{kpc}$, yet the mass enclosed at $R<2.5 \mathrm{kpc}$ weighs in the total gravitational strength, though it does not represent a leading term.

However, adopting the values of $\rho_{0}$ determined from the RC analysis can be seen as imposing a continuity of the DM profile at the boundary between the two regions. We highlight the range of $\rho_{0}$ allowed by the latter, in Fig. 4, so that it can act as a possible prior in the determination of a favorite range of allowed $\gamma$. We summarize the content of Fig. 4 in the Tables 1-5, where we list the stellar mass content (and its uncertainty) and the estimated DM mass for all of our bulge and disc components. We also report the range of $\gamma$ allowed, if one imposes a prior on the local DM density, as discussed above.

Interestingly, it is to be noticed that the allowed DM mass estimated with this method is compatible with zero (unsurprisingly, given that we do expect baryons to dominate the DM potential in such a small central region) for almost our entire sample of morphologies. Yet, even with the quite sizable uncertainties at hand, it is possible to put a sound upper (and in few cases, lower) limit to the estimate of the index $\gamma$, locally for this inner region of the MW.

\section{Conclusions}

We have presented an analysis of the DM density profile in the Galactic bulge region, following and complementing upon the recent analysis in [17]. By adopting an observational estimate of the 


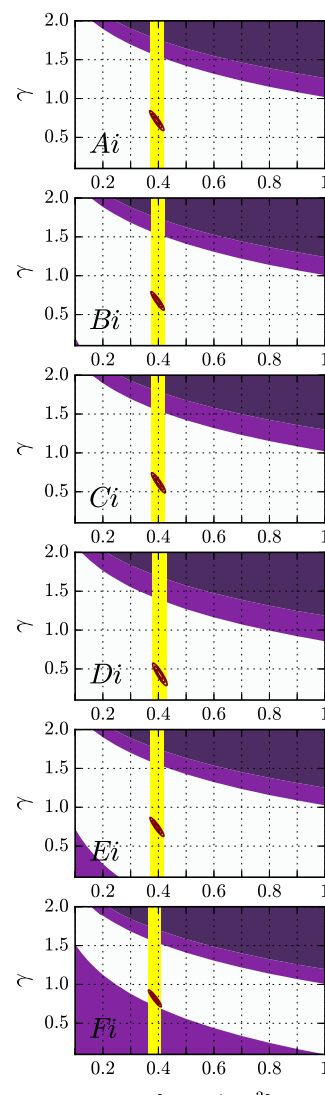

$\rho_{0}\left[\mathrm{GeV} / \mathrm{cm}^{3}\right]$

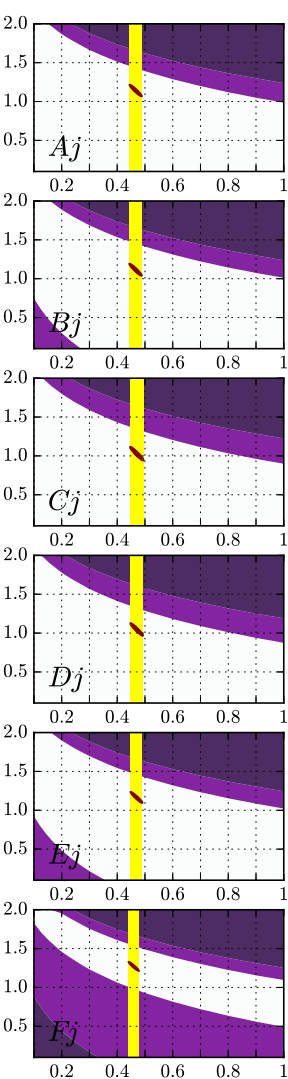

$\rho_{0}\left[\mathrm{GeV} / \mathrm{cm}^{3}\right]$

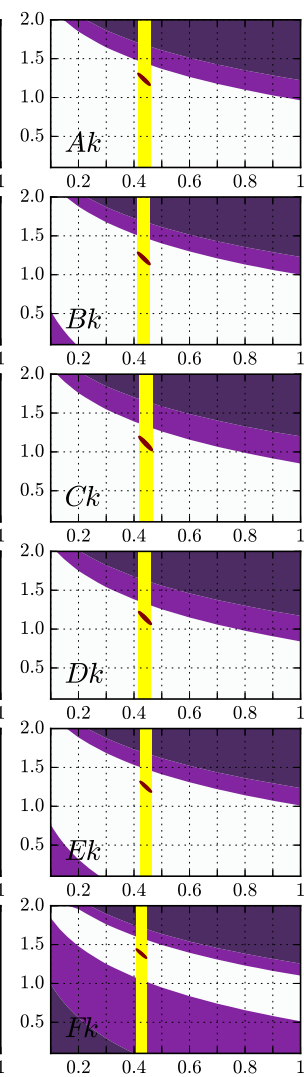

$\rho_{0}\left[\mathrm{GeV} / \mathrm{cm}^{3}\right]$

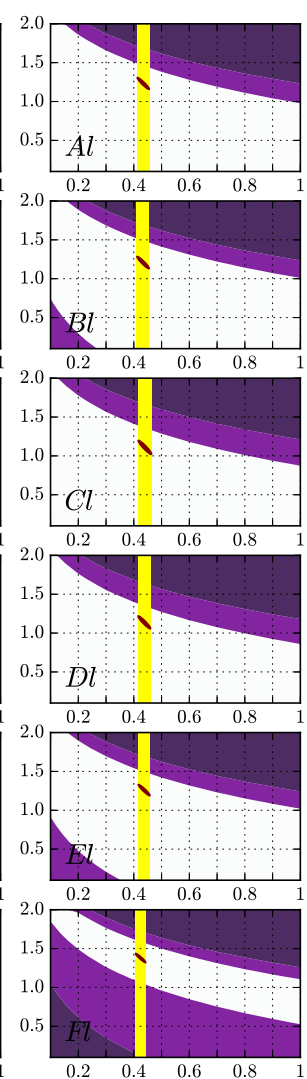

$\rho_{0}\left[\mathrm{GeV} / \mathrm{cm}^{3}\right]$

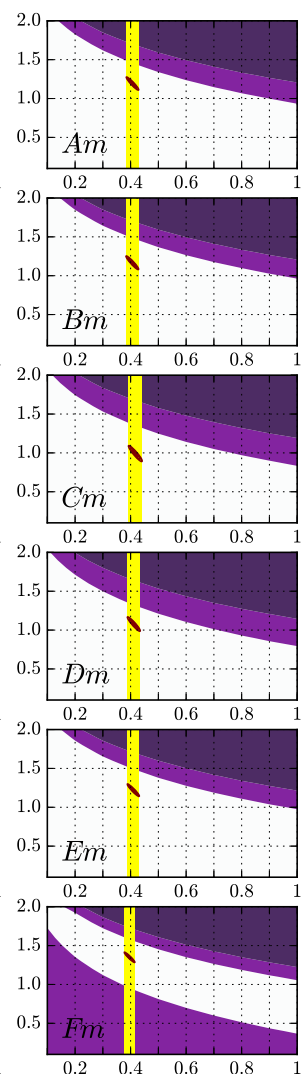

$\rho_{0}\left[\mathrm{GeV} / \mathrm{cm}^{3}\right]$

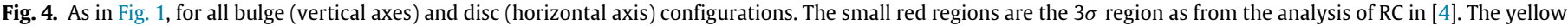

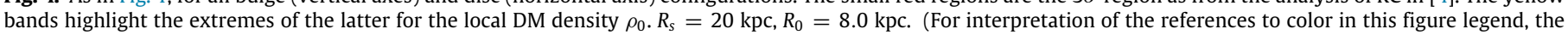
reader is referred to the web version of this article.)

Table 1

Values of stellar mass and allowed DM mass for all bulge configurations. The value of index $\gamma$, as allowed at $1 \sigma$ within the local DM density $\rho_{0}$ band identified by the RC analysis (yellow line in Fig. 4. Stellar disc [7] (" $i$ "), $M_{*}^{\text {disc }}=(1.06 \pm$ $0.11) \times 10^{10} M_{\odot} ; R_{S}=20 \mathrm{kpc}, R_{0}=8.0 \mathrm{kpc}$.

\begin{tabular}{llllll}
\hline Bulge & Reference & $M_{*}^{\text {bulge }}\left(\times 10^{10} M_{\odot}\right)$ & $M_{*}\left(\times 10^{10} M_{\odot}\right)$ & $M_{D M}\left(\times 10^{9} M_{\odot}\right)$ & $\gamma$ \\
\hline A & {$[27](G 2)$} & $0.4 \pm 0.4$ & $1.4 \pm 0.4$ & $4 \pm 4$ & $0.00-1.59$ \\
B & {$[27](E 2)$} & $0.4 \pm 0.4$ & $1.4 \pm 0.4$ & $4 \pm 4$ & $0.00-1.57$ \\
C & {$[28]$} & $0.5 \pm 0.5$ & $1.5 \pm 0.5$ & $3 \pm 5$ & $0.00-1.58$ \\
D & {$[29]$} & $0.6 \pm 0.4$ & $1.7 \pm 0.5$ & $2 \pm 5$ & $0.00-1.43$ \\
E & {$[30]$} & $0.3 \pm 0.4$ & $1.4 \pm 0.4$ & $4 \pm 4$ & $0.00-1.59$ \\
F & {$[31]$} & $0.3 \pm 0.3$ & $1.3 \pm 0.3$ & $5 \pm 3$ & $0.69-1.58$ \\
\hline
\end{tabular}

Table 2

Values of stellar mass and allowed DM mass for all bulge configurations. The value of index $\gamma$, as allowed at $1 \sigma$ within the local DM density $\rho_{0}$ band identified by the RC analysis (yellow line in Fig. 4. Stellar disc [26] ("j"), $M_{*}^{\text {disc }}=(0.55 \pm$ $0.06) \times 10^{10} M_{\odot} ; R_{S}=20 \mathrm{kpc}, R_{0}=8.0 \mathrm{kpc}$.

\begin{tabular}{llllll}
\hline Bulge & Reference & $M_{*}^{\text {bulge }}\left(\times 10^{10} M_{\odot}\right)$ & $M_{*}\left(\times 10^{10} M_{\odot}\right)$ & $M_{D M}\left(\times 10^{9} M_{\odot}\right)$ & $\gamma$ \\
\hline A & {$[27](G 2)$} & $0.9 \pm 0.4$ & $1.5 \pm 0.4$ & $4 \pm 4$ & $0.00-1.46$ \\
B & {$[27](E 2)$} & $0.9 \pm 0.4$ & $1.4 \pm 0.4$ & $4 \pm 4$ & $0.00-1.48$ \\
C & {$[28]$} & $1.1 \pm 0.5$ & $1.7 \pm 0.5$ & $2 \pm 5$ & $0.00-1.38$ \\
D & {$[29]$} & $1.1 \pm 0.4$ & $1.6 \pm 0.4$ & $2 \pm 5$ & $0.00-1.35$ \\
E & {$[30]$} & $0.8 \pm 0.4$ & $1.4 \pm 0.4$ & $5 \pm 4$ & $0.00-1.49$ \\
F & {$[31]$} & $0.6 \pm 0.3$ & $1.2 \pm 0.3$ & $6 \pm 3$ & $0.96-1.56$ \\
\hline
\end{tabular}

total dynamical mass, and a large array of observationally-derived morphologies for the stellar components, we are able to estimate the current statistical uncertainties on the allowed DM mass. We find it to vary between $6 \%$ and $45 \%$ the total dynamical mass. These figures are compatible with those of the analysis in [17], yet they define a bigger range (compatible with zero at one sigma) as a consequence of the systematic arising from our ignorance on the actual morphology of the region of the bulge, and of the statistical on the actual normalization of the visible component.

Both type of uncertainties are sizable and mask away sound, general conclusions on the preferred region of the parameter space (if the DM mass is fit to a gNFW profile): whereas cuspy profiles are not strictly prohibited (although indexes $\gamma \gtrsim 1.5$ are generally disfavored at $1 \sigma$ at least, depending on the bulge/disc configuration), 
Table 3

Values of stellar mass and allowed DM mass for all bulge configurations. The value of index $\gamma$, as allowed at $1 \sigma$ within the local DM density $\rho_{0}$ band identified by the RC analysis (yellow line in Fig. 4. Stellar disc [32] ("k"), $M_{*}^{\text {disc }}=(0.93 \pm$ $0.10) \times 10^{10} M_{\odot} ; R_{s}=20 \mathrm{kpc}, R_{0}=8.0 \mathrm{kpc}$.

\begin{tabular}{llllll}
\hline Bulge & Reference & $M_{*}^{\text {bulge }}\left(\times 10^{10} M_{\odot}\right)$ & $M_{*}\left(\times 10^{10} M_{\odot}\right)$ & $M_{D M}\left(\times 10^{9} M_{\odot}\right)$ & $\gamma$ \\
\hline A & {$[27](G 2)$} & $0.2 \pm 0.4$ & $1.1 \pm 0.4$ & $7 \pm 4$ & $1.28-2.00$ \\
B & {$[27](E 2)$} & $0.2 \pm 0.4$ & $1.1 \pm 0.4$ & $7 \pm 4$ & $1.34-2.00$ \\
C & {$[28]$} & $0.2 \pm 0.5$ & $1.2 \pm 0.5$ & $5 \pm 5$ & $0.96-2.00$ \\
D & {$[29]$} & $0.4 \pm 0.4$ & $1.3 \pm 0.5$ & $7 \pm 4$ & $0.46-2.00$ \\
E & {$[30]$} & $0.2 \pm 0.4$ & $1.1 \pm 0.4$ & $8 \pm 3$ & $1.38-2.00$ \\
F & {$[31]$} & $0.1 \pm 0.3$ & $1.1 \pm 0.3$ & $1.55-2.00$ \\
\hline
\end{tabular}

Table 4

Values of stellar mass and allowed DM mass for all bulge configurations. The value of index $\gamma$, as allowed at $1 \sigma$ within the local DM density $\rho_{0}$ band identified by the RC analysis (yellow line in Fig. 4. Stellar disc [33] ("l"), $M_{*}^{\text {disc }}=(0.48 \pm$ $0.05) \times 10^{10} M_{\odot} ; R_{S}=20 \mathrm{kpc}, R_{0}=8.0 \mathrm{kpc}$.

\begin{tabular}{llllll}
\hline Bulge & Reference & $M_{*}^{\text {bulge }}\left(\times 10^{10} M_{\odot}\right)$ & $M_{*}\left(\times 10^{10} M_{\odot}\right)$ & $M_{D M}\left(\times 10^{9} M_{\odot}\right)$ & $\gamma$ \\
\hline A & {$[27](G 2)$} & $1.0 \pm 0.4$ & $1.5 \pm 0.4$ & $4 \pm 4$ & $0.00-1.49$ \\
B & {$[27](E 2)$} & $0.9 \pm 0.4$ & $1.4 \pm 0.4$ & $4 \pm 4$ & $0.00-1.52$ \\
C & {$[28]$} & $1.2 \pm 0.5$ & $1.7 \pm 0.5$ & $2 \pm 5$ & $0.00-1.39$ \\
D & {$[29]$} & $1.2 \pm 0.4$ & $1.7 \pm 0.4$ & $2 \pm 4$ & $0.00-1.38$ \\
E & {$[30]$} & $0.9 \pm 0.4$ & $1.4 \pm 0.4$ & $4 \pm 4$ & $0.00-1.52$ \\
F & {$[31]$} & $0.7 \pm 0.3$ & $1.2 \pm 0.3$ & $7 \pm 3$ & $1.04-1.61$ \\
\hline
\end{tabular}

Table 5

Values of stellar mass and allowed DM mass for all bulge configurations. The value of index $\gamma$, as allowed at $1 \sigma$ within the local DM density $\rho_{0}$ band identified by the RC analysis (yellow line in Fig. 4 . Stellar disc [34] (" $m$ "), $M_{*}^{\text {disc }}=(0.60 \pm$ $0.06) \times 10^{10} M_{\odot} ; R_{S}=20 \mathrm{kpc}, R_{0}=8.0 \mathrm{kpc}$.

\begin{tabular}{llllll}
\hline Bulge & Reference & $M_{*}^{\text {bulge }}\left(\times 10^{10} M_{\odot}\right)$ & $M_{*}\left(\times 10^{10} M_{\odot}\right)$ & $M_{D M}\left(\times 10^{9} M_{\odot}\right)$ & $\gamma$ \\
\hline A & {$[27](G 2)$} & $0.9 \pm 0.4$ & $1.5 \pm 0.4$ & $3 \pm 4$ & $0.00-1.49$ \\
B & {$[27](E 2)$} & $0.9 \pm 0.4$ & $1.5 \pm 0.5$ & $4 \pm 4$ & $0.00-1.52$ \\
C & {$[28]$} & $1.1 \pm 0.5$ & $1.7 \pm 0.5$ & $1 \pm 5$ & $0.00-1.39$ \\
D & {$[29]$} & $1.1 \pm 0.4$ & $1.7 \pm 0.4$ & $1 \pm 5$ & $0.00-1.35$ \\
E & {$[30]$} & $0.8 \pm 0.4$ & $1.5 \pm 0.4$ & $4 \pm 4$ & $0.00-1.52$ \\
F & {$[31]$} & $0.6 \pm 0.3$ & $1.3 \pm 0.3$ & $6 \pm 3$ & $0.99-1.61$ \\
\hline
\end{tabular}

neither are flat profiles, an index $\gamma=0$ allowed for many of the morphologies at study.

These results are compatible with analysis of outer regions, at galactocentric radii $R>2.5 \mathrm{kpc}$, performed with global techniques based on a fitting of the Rotation Curve, but this is hardly indicative, nor surprising, given the very large degree of uncertainty described above.

We argue that it is still early for a conclusive answer about the distribution of DM in the Galactic center, and that the bottleneck of the current impasse is the estimate of the visible component morphology and normalization, rather than that on the total dynamical mass, in a similar fashion to what happens for the determination of the DM profile in the outer regions of the Galaxy.

Future surveys dedicated to the stellar census and dynamics in that region, like the forthcoming Wide Field InfraRed Survey Telescope (WFIRST) will help in the endeavor of reducing uncertainties, and move toward sounder conclusions about this fascinating problem.

\section{Acknowledgments}

We thank G. Bertone, F. Calore, M. Pato, and P.D. Serpico for valuable comments on this manuscript. M.B. thanks M. Peiró for valuable help on numerical recipes.

The authors would like to express a special thanks to the Mainz Institute for Theoretical Physics (MITP) for its hospitality and support.

F.I. is supported by FAPESP JP project 2014/11070-2.

\section{References}

[1] R. Catena, P. Ullio, A novel determination of the local dark matter density, J. Cosmol. Astropart. Phys. 1008 (2010) 004. http://dx.doi.org/10.1088/14757516/2010/08/004. arXiv:0907.0018.
[2] F. Iocco, M. Pato, G. Bertone, P. Jetzer, Dark matter distribution in the Milky Way: microlensing and dynamical constraints, J. Cosmol. Astropart. Phys. 1111 (2011) 029. http://dx.doi.org/10.1088/1475-7516/2011/11/029. arXiv: 1107.5810 .

[3] F. Nesti, P. Salucci, The dark matter halo of the Milky Way, AD 2013, J. Cosmol. Astropart. Phys. 1307 (2013) 016. http://dx.doi.org/10.1088/1475-7516/2013/ 07/016. arXiv:1304.5127.

[4] M. Pato, F. Iocco, G. Bertone, Dynamical constraints on the dark matter distribution in the Milky Way, J. Cosmol. Astropart. Phys. 1512 (12) (2015) 001. http://dx.doi.org/10.1088/1475-7516/2015/12/001. arXiv:1504.06324.

[5] J.I. Read, The local dark matter density, J. Phys. G: Nucl. Phys. 41 (6) (2014) 063101. http://dx.doi.org/10.1088/0954-3899/41/6/063101.arXiv:1404.1938.

[6] L. Chemin, F. Renaud, C. Soubiran, Incorrect rotation curve of the Milky Way, Astron. Astrophys. 578 (2015) A14. http://dx.doi.org/10.1051/00046361/201526040. arXiv:1504.01507.

[7] J. Bovy, H.-W. Rix, A direct dynamical measurement of the Milky Way's disk surface density profile, disk scale length, and dark matter profile at $4 \mathrm{kpc} \lesssim$ $R \lesssim 9$ kpc, Astrophys. J. 779 (2013) 115. http://dx.doi.org/10.1088/0004637X/779/2/115. arXiv:1309.0809.

[8] M. Pato, F. Iocco, The dark matter profile of the Milky Way: a non-parametric reconstruction, Astrophys. J. 803 (1) (2015) L3. http://dx.doi.org/10.1088/ 2041-8205/803/1/L3. arXiv:1504.03317.

[9] W.J.G. de Blok, The core-cusp problem, Adv. Astron. 2010 (2010) 789293. http://dx.doi.org/10.1155/2010/789293. arXiv:0910.3538.

[10] F. Governato, C. Brook, L. Mayer, A. Brooks, G. Rhee, J. Wadsley, P. Jonsson, B. Willman, G. Stinson, T. Quinn, P. Madau, Bulgeless dwarf galaxies and dark matter cores from supernova-driven outflows, Nature 463 (2010) 203-206. http://dx.doi.org/10.1038/nature08640. arXiv:0911.2237.

[11] J. Oñorbe, M. Boylan-Kolchin, J.S. Bullock, P.F. Hopkins, D. Kereš, C.-A. FaucherGiguère, E. Quataert, N. Murray, Forged in FIRE: cusps, cores and baryons in low-mass dwarf galaxies, Mon. Not. R. Astron. Soc. 454 (2015) 2092-2106. http://dx.doi.org/10.1093/mnras/stv2072. arXiv:1502.02036.

[12] E. Tollet, A.V. Macciò, A.A. Dutton, G.S. Stinson, L. Wang, C. Penzo, T.A. Gutcke, T. Buck, X. Kang, C. Brook, A. Di Cintio, B.W. Keller, J. Wadsley, NIHAO-IV: core creation and destruction in dark matter density profiles across cosmic time, Mon. Not. R. Astron. Soc. 456 (2016) 3542-3552. http://dx.doi.org/10.1093/ mnras/stv2856. arXiv:1507.03590.

[13] F. Governato, A. Zolotov, A. Pontzen, C. Christensen, S.H. Oh, A.M. Brooks, T. Quinn, S. Shen, J. Wadsley, Cuspy no more: how outflows affect the central 
dark matter and baryon distribution in $\Lambda$ cold dark matter galaxies, Mon. Not. R. Astron. Soc. 422 (2012) 1231-1240. http://dx.doi.org/10.1111/j.13652966.2012.20696.x. arXiv: 1202.0554.

[14] A. Di Cintio, C.B. Brook, A.V. Macci, G.S. Stinson, A. Knebe, A.A. Dutton, J. Wadsley, The dependence of dark matter profiles on the stellar-to-halo mass ratio: a prediction for cusps versus cores, Mon. Not. R. Astron. Soc. 437 (1) (2014) 415-423. http://dx.doi.org/10.1093/mnras/stt1891. arXiv:1306.0898.

[15] F. Calore, N. Bozorgnia, M. Lovell, G. Bertone, M. Schaller, C.S. Frenk, R.A. Crain, J. Schaye, T. Theuns, J.W. Trayford, Simulated Milky Way analogues: implications for dark matter indirect searches, J. Cosmol. Astropart. Phys. 1512 (12) (2015) 053. http://dx.doi.org/10.1088/1475-7516/2015/12/053. arXiv:1509.02164.

[16] M. Schaller, et al., Dark matter annihilation radiation in hydrodynamic simulations of Milky Way haloes, Mon. Not. R. Astron. Soc. 455 (4) (2016) 4442-4451. http://dx.doi.org/10.1093/mnras/stv2667. arXiv:1509.02166.

[17] D. Hooper, The density of dark matter in the galactic bulge and implications for indirect detection, J. Cosmol. Astropart. Phys. (2016) (submitted for publication) arXiv: 1608.00003

[18] M. Portail, C. Wegg, O. Gerhard, I. Martinez-Valpuesta, Made-to-measure models of the galactic box/peanut bulge: stellar and total mass in the bulge region, Mon. Not. R. Astron. Soc. 448 (1) (2015) 713-731.

[19] M. Portail, O. Gerhard, C. Wegg, M. Ness, Dynamical modelling of the galactic bulge and bar: Pattern speed, stellar, and dark matter mass distributions (2016). arXiv:1608.07954.

[20] C.D. Howard, R.M. Rich, D.B. Reitzel, A. Koch, R. De Propris, H. Zhao, The bulge radial velocity assay (BRAVA): I. Sample selection and a rotation curve, Astrophys. J. 688 (2008) 1060. http://dx.doi.org/10.1086/592106. arXiv:0807. 3967.

[21] A. Kunder, A. Koch, R.M. Rich, R. de Propris, C.D. Howard, S.A. Stubbs, C.I Johnson, J. Shen, Y. Wang, A.C. Robin, J. Kormendy, M. Soto, P. Frinchaboy, D.B. Reitzel, H. Zhao, L. Origlia, The bulge radial velocity assay (BRAVA). II. Complete sample and data release, Astron. J. 143 (2012) 57. http://dx.doi.org/10.1088/ 0004-6256/143/3/57. arXiv:1112.1955.

[22] F. Iocco, M. Pato, G. Bertone, Evidence for dark matter in the inner Milky Way, Nat. Phys. 11 (2015) 245-248. http://dx.doi.org/10.1038/nphys3237. arXiv: 1502.03821.

[23] P. Popowski, et al., Microlensing optical depth towards the galactic bulge using clump giants from the MACHO survey, Astrophys. J. 631 (2005) 879-905. http://dx.doi.org/10.1086/432246. arXiv:astro-ph/0410319.
[24] K. Ferrière, Global model of the interstellar medium in our galaxy with new constraints on the hot gas component, Astrophys. J. 497 (2) (1998) 759.

[25] K. Ferriere, W. Gillard, P. Jean, Spatial distribution of interstellar gas in the innermost 3 kpc of our Galaxy, Astron. Astrophys. 467 (2007) 611-627. http: //dx.doi.org/10.1051/0004-6361:20066992. arXiv:astro-ph/0702532.

[26] C. Han, A.P. Gould, Stellar contribution to the Galactic bulge microlensing optical depth, Astrophys. J. 592 (2003) 172-175. http://dx.doi.org/10.1086/ 375706. arXiv:astro-ph/0303309.

[27] E. Dwek, R.G. Arendt, M.G. Hauser, T. Kelsall, C.M. Lisse, S.H. Moseley, R.F. Silverberg, T.J. Sodroski, J.L. Weiland, Morphology, near infrared luminosity, and mass of the galactic bulge from Cobe dirbe observations, Astrophys. J. 445 (1995) 716. http://dx.doi.org/10.1086/175734.

[28] E. Vanhollebeke, M.A.T. Groenewegen, L. Girardi, Stellar populations in the Galactic bulge (2009) arXiv:0903.0946.

[29] N. Bissantz, O. Gerhard, Spiral arms, bar shape and bulge microlensing in the milky way, Mon. Not. R. Astron. Soc. 330 (2002) 591. http://dx.doi.org/10.1046/ j.1365-8711.2002.05116.x. arXiv:astro-ph/0110368.

[30] H. Zhao, A self-consistent dynamical model for the COBE detected galactic bar, Mon. Not. R. Astron. Soc. 283 (1996) 149-166. http://dx.doi.org/10.1093/ mnras/283.1.149. arXiv:astro-ph/9512064.

[31] A.C. Robin, D.J. Marshall, M. Schultheis, C. Reyle, Stellar populations in the Milky Way bulge region : Towards solving the Galactic bulge and bar shapes using 2MASS data, Astron. Astrophys. 538 (2012) A106. http://dx.doi.org/10. 1051/0004-6361/201116512. arXiv:1111.5744.

[32] S. Calchi Novati, L. Mancini, Microlensing towards the LMC: self lensing for OGLE-II and OGLE-III, Mon. Not. R. Astron. Soc. 416 (2011) 1292-1301. http: //dx.doi.org/10.1111/j.1365-2966.2011.19123.x. arXiv:1105.4615.

[33] J.T.A. de Jong, B. Yanny, H.-W. Rix, A.E. Dolphin, N.F. Martin, T.C. Beers, Mapping the stellar structure of the Milky Way thick disk and halo using SEGUE photometry, Astrophys. J. 714 (2010) 663-674. http://dx.doi.org/10.1088/0004637X/714/1/663. arXiv:0911.3900.

[34] M. Juric, et al., The Milky Way tomography with SDSS. 1. Stellar number density distribution, Astrophys. J. 673 (2008) 864-914. http://dx.doi.org/10. 1086/523619. arXiv:astro-ph/0510520. 